\title{
Investigation of Thermal Degradation of Branched Polypropylene via Rheology
}

\author{
Jiri Drabek $^{1, \text { a) }}$ and Martin Zatloukal ${ }^{1, b)}$ \\ ${ }^{1}$ Polymer Centre, Faculty of Technology, Tomas Bata University in Zlin, \\ nam. T. G. Masaryka 275, 76272 Zlin, Czech Republic \\ a)drabek@ft.utb.cz \\ ${ }^{\text {b) }}$ Corresponding author: mzatloukal@ft.utb.cz
}

\begin{abstract}
In this work, virgin as well as thermally degraded branched polypropylenes were investigated by using rotational and Sentmanat extensional rheometers. Based on the shear and extensional rheology data it was deduced that both chain scission and chain branching takes place during thermal degradation of the tested polypropylene. It was found that simple phenomenological constitutive equations such as Generalized Newtonian law and modified White Metzner model can be used to describe the measured steady state shear and uniaxial extensional viscosity data as well as for the simple quantification of the extensional strain hardening via their model parameters.
\end{abstract}

\section{INTRODUCTION}

Thermal degradation of polymers, which can occur during their processing, can significantly influence their molecular structure via chain scission, branching or even crosslinking, which can narrower the processing window as well as to reduce the basic properties of the final product considerably. In order to stabilize the polymer processing, it is very important to understand all possible structural changes of the particular polymer due to thermal degradation. It has been shown that rheology can be used as the powerful tool to understand degradation of different polymers due to its sensitivity to very small changes in the molecular structure of polymers as well as to understand their molecular structure via utilization of advanced constitutive equations, which are based on the molecular arguments [1-10]. With the aim to extend the knowledge in this area, thermal stability of branched polypropylene was investigated in this work by using basic rheological tool and methodologies. Specific attention was paid to quantification of the measured data by using two simple phenomenological constitutive equations.

\section{EXPERIMENTAL}

In this work, branched PP Daploy WB180HMS (Borealis Polyolefine) has been used. In the first step, the polymer was thermally degraded. For such a purpose, polymer was added into a Rosand RH7-2 twin bore capillary rheometer, melted and kept given time ( 3 and 7 hours in this study) at $240^{\circ} \mathrm{C}$. Obtained thermally degraded polymers together with the virgin polymer were consequently pressed to plates from which the final testing samples were prepared. Linear viscoelastic properties were measured on Advanced Rheometric Expansion System (ARES 2000 model, Rheometrics Scientific, USA) at $170^{\circ} \mathrm{C}, 180^{\circ} \mathrm{C}$ and $190^{\circ} \mathrm{C}$ in parallel plates mode. In order to capture Newtonian plateau, shear creep measurements were performed at the reference temperature equal to $180^{\circ} \mathrm{C}$. The measured data were fitted by the multimode Maxwell model to determine discontinuous relaxation spectrum. Transient extensional viscosity, $\eta_{\mathrm{E}}$, was determined at $170^{\circ} \mathrm{C}, 180^{\circ} \mathrm{C}$ and $190^{\circ} \mathrm{C}$ on Sentmanat Extensional Rheometer (SER-HV-A01 model, Xpansion Instruments, USA [11-13]) attached to ARES 2000 rotational rheometer and the obtained measured data were shifted to the reference temperature. The 'steady state' extensional 
viscosity data were taken from the peaks appearing on the transient viscosity curves for corresponding extensional strain rates.

\section{THEORETICAL}

\section{Generalized Newtonian Fluid Model}

In this work, recently proposed generalized Newtonian fluid model has been utilized [14-15]:

$$
\tau=2 \eta\left(I_{|\mathrm{D}|}, I I_{\mathrm{D}}, I I I_{\mathrm{D}}\right) D
$$

where $\tau$ means the extra stress tensor, $D$ represents the deformation rate tensor and $\eta$ stands for the viscosity, which is not constant (as in the case of standard Newtonian law), but it is allowed to vary with the first invariant of the absolute value of deformation rate tensor $I_{|\mathrm{D}|}=\operatorname{tr}(|D|)$, (where $|D|$ is defined as the square root of $D^{2}$ ) as well as on the second $I I_{\mathrm{D}}=2 \operatorname{tr}\left(D^{2}\right)$, and third, $I I I_{\mathrm{D}}=\operatorname{det}(D)$, invariants of $D$ according to Eq. 2

$$
\eta\left(I_{|\mathrm{D}|}, I I_{\mathrm{D}}, I I I_{\mathrm{D}}\right)=A^{1-f\left(I_{|\mathrm{D}|}, I I_{\mathrm{D}}, I I I_{\mathrm{D}}\right)} \eta\left(I I_{\mathrm{D}}\right)^{f\left(I_{|\mathrm{D}|}, I I_{\mathrm{D}}, I I I_{\mathrm{D}}\right)}
$$

where $\eta\left(I I_{\mathrm{D}}\right)$ is given by the well-known Carreau-Yasuda model, Eq. 3 and $f\left(I_{|\mathrm{D}|}, I I_{\mathrm{D}}, I I I_{\mathrm{D}}\right)$ is given by Eq. 4

$$
\begin{gathered}
\eta\left(I I_{\mathrm{D}}\right)=\frac{\eta_{0} a_{\mathrm{T}}}{\left[1+\left(\lambda a_{\mathrm{T}} \sqrt{I I_{\mathrm{D}}}\right)^{a}\right]^{\left(\frac{1-n}{a}\right)}} \\
f\left(I_{|\mathrm{D}|}, I I_{\mathrm{D}}, I I I_{\mathrm{D}}\right)=\left\{\tanh \left[\alpha a_{\mathrm{T}}\left(1+\frac{1}{4(\sqrt{3})^{3}}\right)^{-\psi}\left(\left|1+\frac{I I I_{\mathrm{D}}}{I I_{\mathrm{D}}^{3 / 2}}\right|\right)^{\psi} \frac{\sqrt[3]{4\left|I I I_{\mathrm{D}}\right|}+I_{|\mathrm{D}|}}{3}+\beta\right] \frac{1}{\tanh (\beta)}\right\}^{\zeta}
\end{gathered}
$$

here $A, \eta_{0}, \lambda, a, n, \alpha, \psi, \beta, \zeta$ are adjustable parameters and $a_{\mathrm{T}}$ is temperature shift factor.

\section{Modified White-Metzner Model}

Modified White-Metzner model constitutive equation is a simple Maxwell model for which the viscosity and relaxation time are allowed to vary with the second invariant of the strain rate deformation tensor [16]. It takes the following form:

$$
\tau+\lambda\left(I I_{\mathrm{D}}\right) \tau=2 \eta\left(I I_{\mathrm{D}}\right) D
$$

where $\tau$ is the upper convected time derivative of stress tensor, $D$ is the deformation rate tensor, $I I_{\mathrm{D}}$ is the second invariant of the rate of deformation tensor, $\lambda\left(I I_{\mathrm{D}}\right)$ stands for the deformation rate-dependent relaxation time and $\eta\left(I I_{\mathrm{D}}\right)$ is the deformation rate-dependent viscosity. Although this modification improves the behaviour in steady shear flows, in elongational flows the model predicts unrealistic infinite elongational viscosity. This problem was overcome by Barnes and Roberts [16], who showed that, for specific functions of $\lambda\left(I I_{\mathrm{D}}\right)$ and $\eta\left(I I_{\mathrm{D}}\right)$ with $\left(\lambda_{0} / K_{2}\right)<$ $(\sqrt{3} / 2)$ (see Eqs. 3 and 6 ), the model does not predict infinite elongational viscosity and can be used for a very good description of elongational viscosity of a wide range of real polymer melts: 


$$
\lambda\left(I I_{\mathrm{D}}\right)=\frac{\lambda_{0} a_{T}}{1+K_{2} a_{T} I I_{\mathrm{D}}}
$$

where $\lambda_{0}$ and $K_{2}$ are constants. Eqs. 3 and 6 together with the physical constraint for $\lambda_{0}$ and $K_{2}$ mentioned above represent the modified White-Metzner model.

\section{RESULTS AND DISCUSSION}

All performed rheological measurements are summarized in Figs. 1-3, from which the following conclusions can be formulated. Firstly, it can be considered that the tested polypropylene is thermorheologically simple because all measured points coincide into one single curve in the van Gurp-Palmen plot, which is provided in Fig. 1a. This is typical for the star-like branched polypropylenes but not for the polyethylenes having typically tree-like branching and thermorheologically complex behavior [17]. Thermorheological simplicity of the tested polymer sample justifies utilization of the time-temperature superposition principle to generate master curves in this work. Secondly, complex viscosity, Newtonian viscosity and shear elasticity (captured through Tan $\delta$ here) decreases with the degradation time, especially within the first three hours. This indicates occurrence of the chain scission during thermal degradation of the tested polypropylene. Transient extensional viscosity data and corresponding 'steady state' extensional viscosity data normalized by the 3 times Newtonian viscosity (measure of the extensional strain hardening and chain branching [17-18]) are provided in Figs. 2a-d. Linear viscoelasticity envelopes depicted in Figs. 2a-c were calculated from the relaxation spectra summarized in Tab. 1.

TABLE 1. Maxwell model parameters for all tested polymer samples at $T=180^{\circ} \mathrm{C}$.

\begin{tabular}{ccccccc}
\hline \multicolumn{4}{c}{ Virgin PP } & \multicolumn{2}{c}{ 3h Degraded PP } & \multicolumn{2}{c}{ 7h Degraded PP } \\
$\mathbf{i}$ & $\boldsymbol{\lambda}_{\mathbf{i}}(\mathbf{s})$ & $\boldsymbol{G}_{\mathbf{i}}(\mathbf{P a})$ & $\boldsymbol{\lambda}_{\mathbf{i}}(\mathbf{s})$ & $\boldsymbol{G}_{\mathbf{i}}(\mathbf{P a})$ & $\boldsymbol{\lambda}_{\mathbf{i}}(\mathbf{s})$ & $\boldsymbol{G}_{\mathbf{i}}(\mathbf{P a})$ \\
\hline 1 & 0.00677 & 52148.6 & 0.00732 & 48414.1 & 0.00744 & 47463.2 \\
2 & 0.03283 & 12150.5 & 0.03517 & 9926.72 & 0.03567 & 9680.62 \\
3 & 0.15912 & 7033.90 & 0.16899 & 5688.47 & 0.17111 & 5616.08 \\
4 & 0.77125 & 2521.01 & 0.81207 & 1914.91 & 0.82079 & 1848.84 \\
5 & 3.73822 & 898.698 & 3.90237 & 686.725 & 3.93727 & 712.969 \\
6 & 18.1189 & 264.527 & 18.7526 & 189.403 & 18.8867 & 144.403 \\
7 & 87.8211 & 31.1191 & 90.1149 & 11.2843 & 90.5977 & 11.1562 \\
8 & 425.663 & 3.85780 & 433.043 & 1.39873 & 434.588 & 1.16212 \\
9 & 2063.16 & 0.28283 & 2080.97 & 0.02156 & 2084.68 & 0.04436 \\
10 & 10000 & 0.03582 & 10000 & 0.01270 & 10000 & 0.00926 \\
\hline
\end{tabular}

Interestingly, the level of the branching firstly increases within first 3 hours of thermal degradation and then decreases as deduced from the measure of the extensional strain hardening provided in Fig. 2d. This indicates presence of the recombination reactions between the polypropylene chains enhancing the branching level, especially within the first 3 hours of thermal degradation.

In the next step, considering validity of the Cox-Merz rule, deformation rate dependent shear and extensional viscosities were fitted by the Generalized Newtonin law (Eqs. 1-4) and modified White Metzner model (Eqs. 5-6). All model parameters are summarized in Tabs. 2-3.

TABLE 2. Generalized Newtonian model parameters for $T=180^{\circ} \mathrm{C}, \psi=8$.

\begin{tabular}{lcccccccc}
\hline Sample name & $\boldsymbol{\eta}_{\mathbf{0}}$ (Pa.s) & $\boldsymbol{\lambda}(\mathbf{s})$ & $\boldsymbol{a}(-)$ & $\boldsymbol{n}(-)$ & $\boldsymbol{\alpha}(\mathbf{s})$ & $\boldsymbol{\beta}(-)$ & $\boldsymbol{\zeta}(-)$ & $\boldsymbol{A}(\mathbf{P a . s})$ \\
\hline Virgin PP & 17413.56 & 9.462 & 0.5252 & 0.4718 & 10.64815 & 0.011488 & 0.015880 & $1.86 \cdot 10^{-16}$ \\
\hline 3h Degraded PP & 11215.75 & 8.691 & 0.6462 & 0.5142 & 12.75510 & 0.022095 & 0.018715 & $2.04 \cdot 10^{-16}$ \\
\hline 7h Degraded PP & 10408.44 & 7.350 & 0.6651 & 0.5108 & 4.256025 & 0.004785 & 0.012814 & $2.06 \cdot 10^{-16}$ \\
\hline
\end{tabular}

TABLE 3. Modified White-Metzner model parameters for $T=180^{\circ} \mathrm{C}$

\begin{tabular}{lcccccc}
\hline Sample name & $\boldsymbol{\eta}_{\mathbf{0}}(\mathbf{P a . s})$ & $\boldsymbol{\lambda}(\mathbf{s})$ & $\boldsymbol{a}(-)$ & $\boldsymbol{n}(-)$ & $\boldsymbol{\lambda}_{\mathbf{0}}(\mathbf{s})$ & $\boldsymbol{\lambda}_{\mathbf{0}} / \boldsymbol{K}_{\mathbf{2}}$ \\
\hline Virgin PP & 17413.56 & 9.462 & 0.5252 & 0.4718 & 462.91 & 0.844665 \\
\hline 3h Degraded PP & 11215.75 & 8.691 & 0.6462 & 0.5142 & 413.35 & 0.845383 \\
\hline 7h Degraded PP & 10408.44 & 7.350 & 0.6651 & 0.5108 & 257.23 & 0.840236 \\
\hline
\end{tabular}


a)

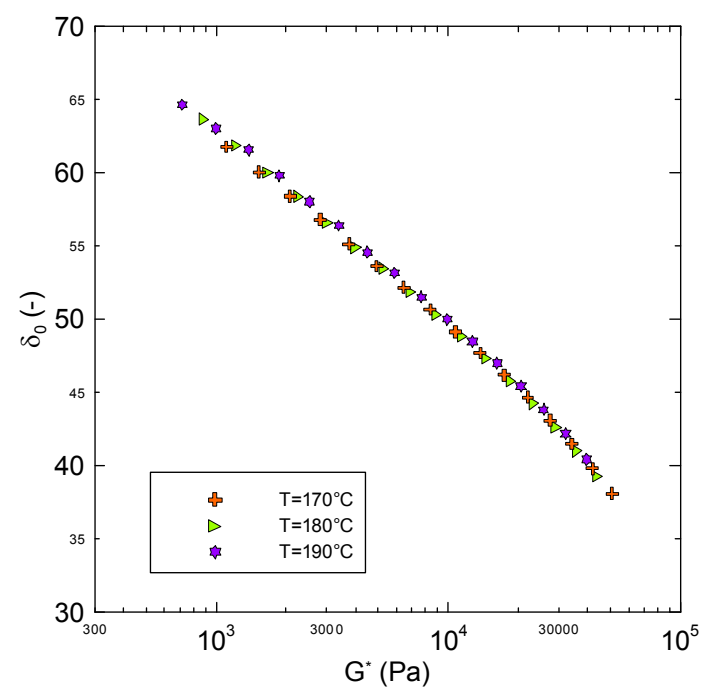

c)

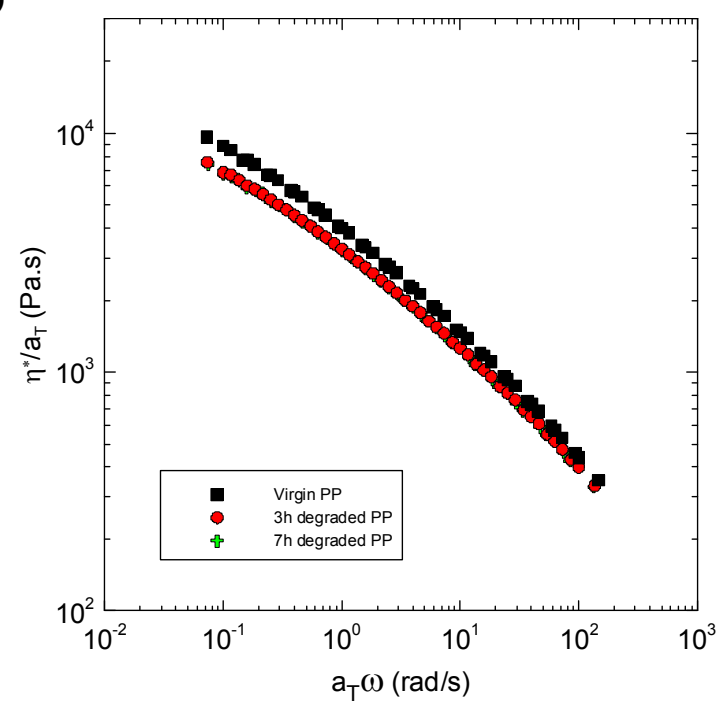

b)

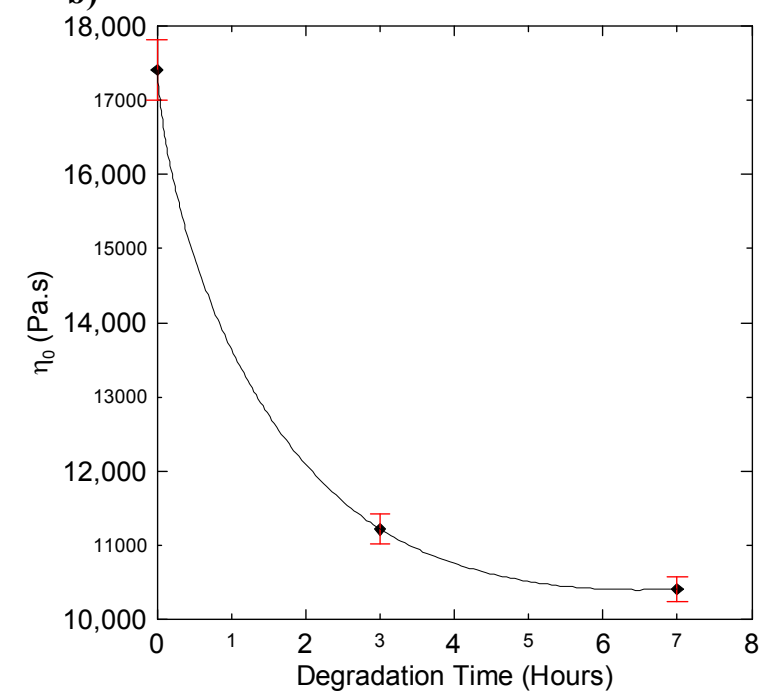

d)

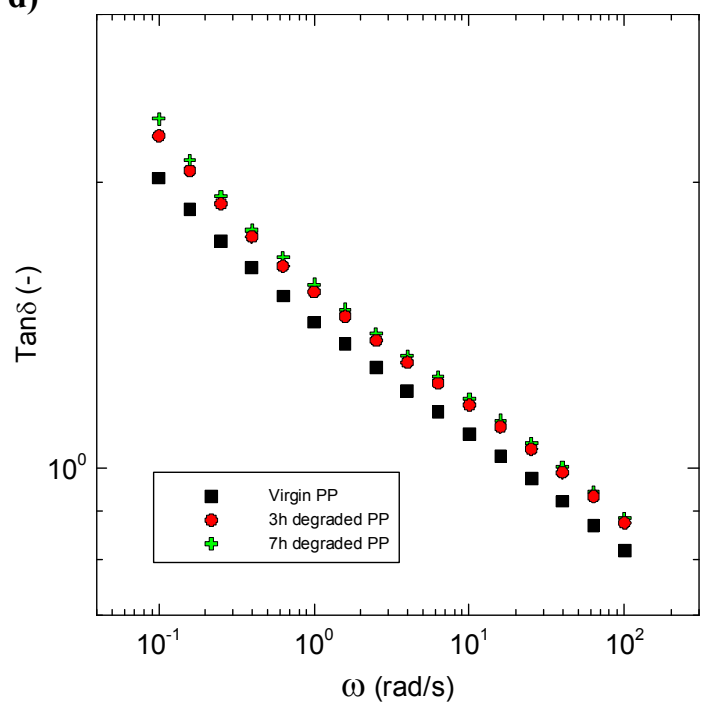

FIGURE 1. Shear rheology of virgin as well as degraded branched polypropylene obtained from rotational rheometer for $180^{\circ} \mathrm{C}$

1a) Van Gurp-Palmen plot, 1b) Newtonian viscosity $\eta_{0}$, 1c) Frequency dependent complex viscosity $\eta^{*}$, 1d) Frequency dependent Tan $\delta$. 
a)

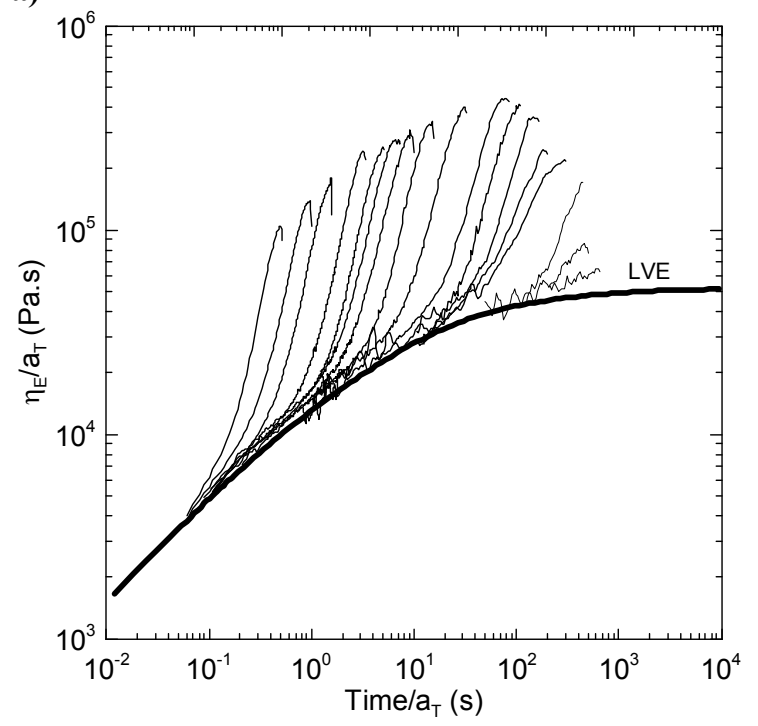

c)

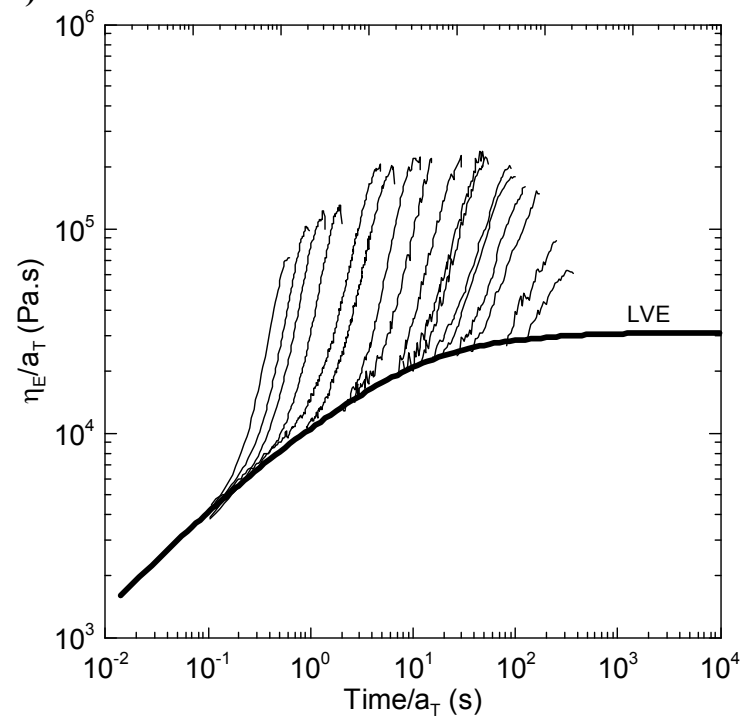

b)

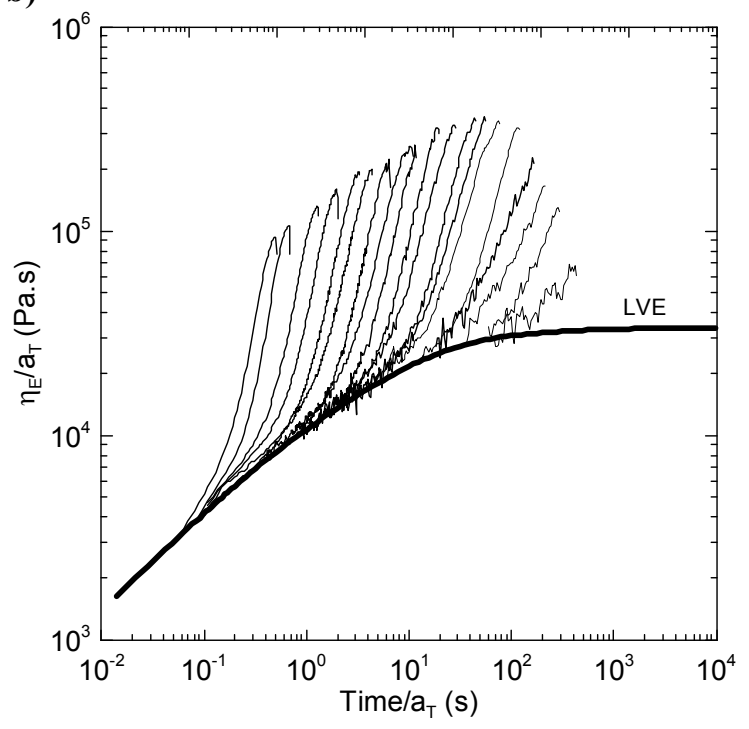

d)

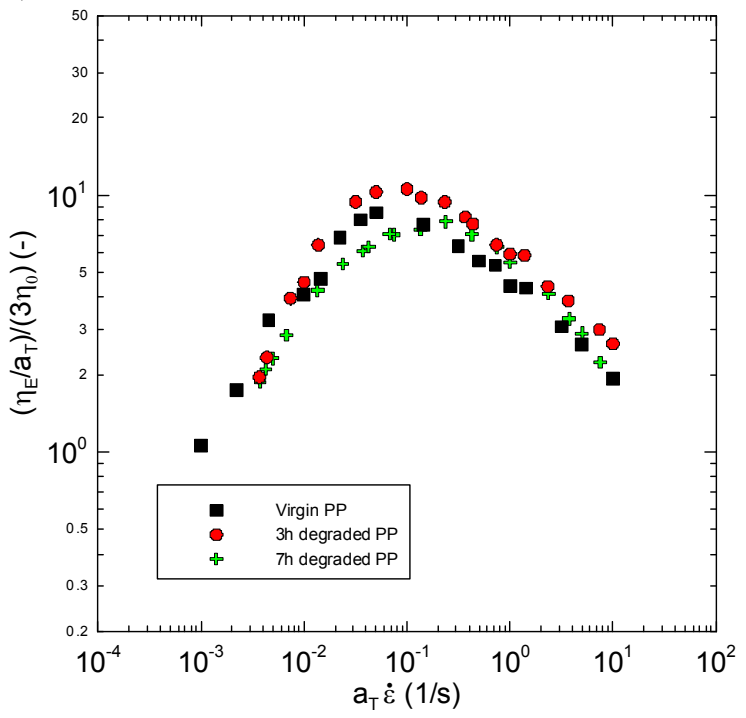

FIGURE 2. Transient and steady state extensional rheology of virgin as well as degraded branched polypropylene obtained from Sentmanat extensional rheometer for $180^{\circ} \mathrm{C}$ 2a) Transient extensional viscosity of the virgin branched polypropylene, 2b) Transient extensional viscosity of the 3 hour thermally degraded branched polypropylene, 2c) Transient extensional viscosity of the 7 hour thermally degraded branched polypropylene, 2d) Steady state extensional viscosity data normalized by the 3 times Newtonian viscosity for virgin and thermally degraded branched polypropylenes. 
a)
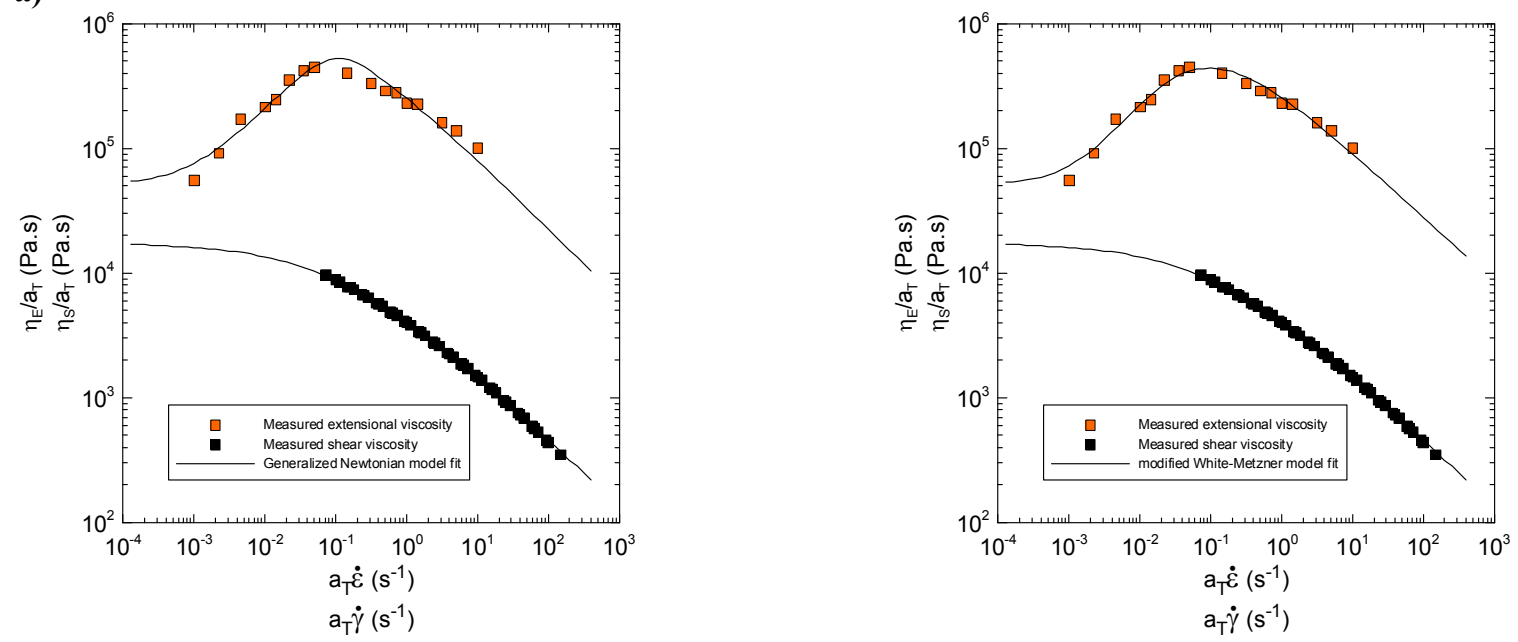

b)
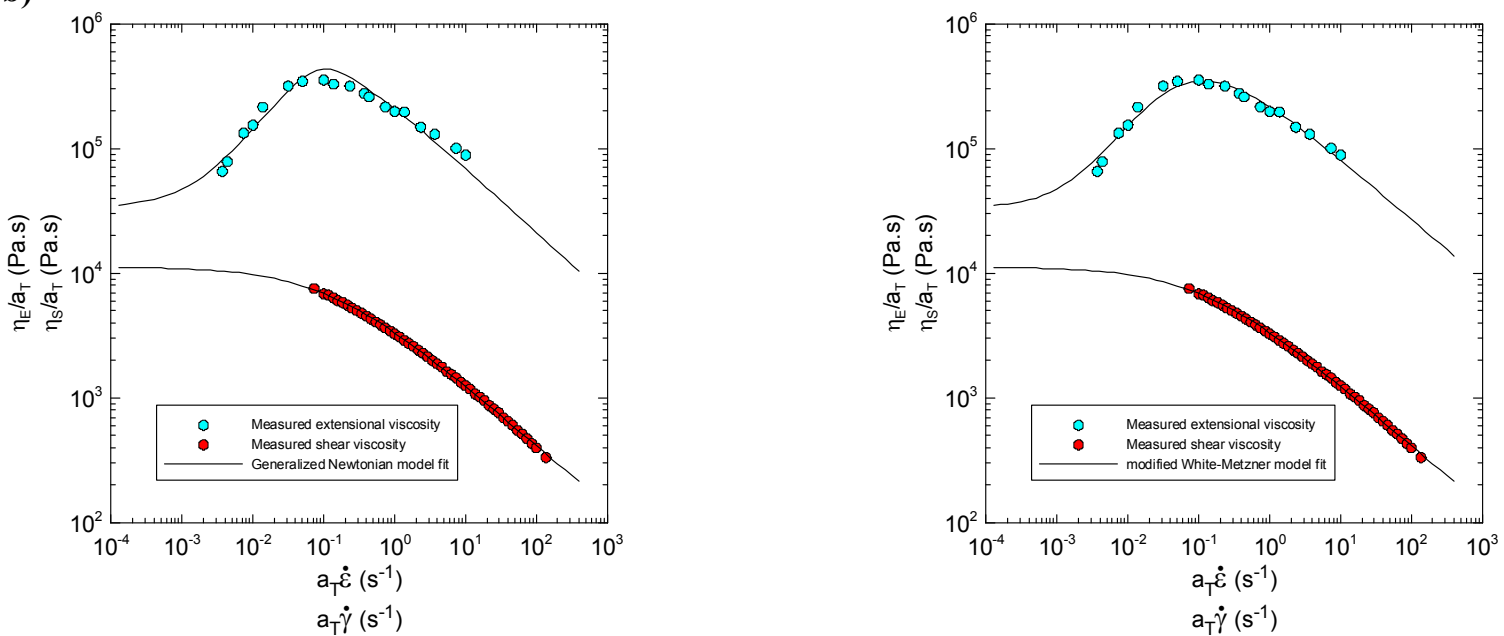

c)
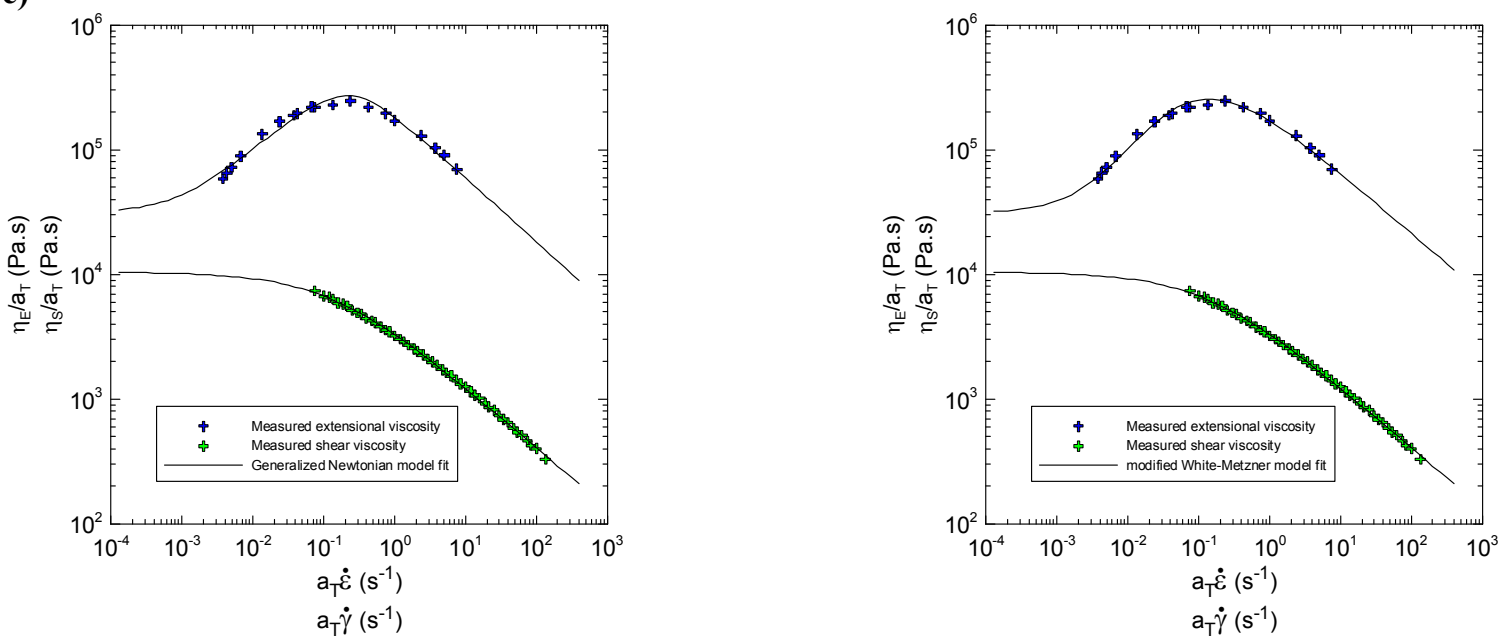

FIGURE 3. Comparison between the measured steady state shear and extensional viscosities and model fits (Left - Generalized Newtonian model, Right - modified White Metzner model) 3a) Virgin branched polypropylene, 3b) 3 hour thermally degraded branched polypropylene, $3 \mathbf{c}) 7$ hour thermally degraded branched polypropylene. 
Comparison between model fitting lines and the measured data are provided in Figs. 3a-c. As can be seen, both models have capability to describe the measured data very well. Importantly, the strain hardening parameter $\zeta$ in the Generalized Newtonian model as well as strain hardening $\lambda_{0} / K_{2}$ parameter in the modified White Metzner model firstly increases with the degradation time and then decreases, i.e. both parameters follows non-monotonic trend in the strain hardening level with respect to degradation time as observed experimentally. This suggests that both models can be used to quantify the level of the branching for polypropylenes via their strain hardening parameters in very simple way due to availability of analytical expressions for steady state shear and extensional viscosities, which makes the experimental data fitting procedure simple and straightforward.

\section{CONCLUSION}

In this work, thermal stability of the branched polypropylene was investigated via shear and extensional viscosity measurements. It was shown that the studied polypropylene is thermorheologically simple showing simultaneous occurrence of chain scission and branching during thermal degradation as deduced from the decreased complex viscosity, Newtonian viscosity and Tan $\delta$ and enhanced extensional strain hardening at low degradation times. It was found that the Generalized Newtonian law as well as modified White Metzner models have capability to firstly, describe the measured steady state shear and extensional rheology of the original and degraded branched polypropylenes and secondly, they can be utilized to quantify the level of extensional strain hardening (i.e. branching level) via their parameters in very simple way.

\section{ACKNOWLEDGMENTS}

This study was supported by the internal grant of TBU in Zlín IGA/FT/2015/013 funded from the resources of specific university research. The author also wishes to acknowledge Joachim Fiebig (Borealis Polyolefine) for donation PP Daploy WB180HMS, which has been used in this work.

\section{REFERENCES}

1. V. H. Rolón-Garrido, M. Kruse and M. H. Wagner, Polym. Deg. Stab. 111, 46-54 (2015).

2. V. H. Rolón-Garrido and M. H. Wagner, Polym. Deg. Stab. 99, 136-145 (2014).

3. V. H. Rolón-Garrido and M. H. Wagner, J. Rheol. 58, 199-222 (2014).

4. V. H. Rolón-Garrido, M. Zatloukal and M. H. Wagner, J. Rheol. 57, 105-129 (2013).

5. M. Kruse, V. H. Rolón-Garrido and M. H. Wagner, AIP Conference Proceedings 1526, 216-229 (2013).

6. J. Dostal, V. Kasparkova, M. Zatloukal, J. Muras and L. Simek, Eur. Polym. J. 44, 2652-2658 (2008).

7. R. Pivokonsky, M. Zatloukal, P. Filip and C. Tzoganakis, J. Non-Newtonian Fluid Mech. 156, 1-6 (2009).

8. H. Jin, J. Gonzalez-Gutierrez, P. Oblak, B. Zupancic and I. Emri, Polym. Deg. Stab. 97, 2262-2272 (2012).

9. J. M. Dealy and R. G. Larson, Structure and rheology of molten polymers: from structure to flow behavior and back again (Hanser Publishers, München, 2006).

10. J. M. Dealy and J. Wang, Melt rheology and its applications in the plastics industry 2nd ed. (Springer, Dordrecht, 2013).

11. M. L. Sentmanat, U.S. Patent No. 6,578,413 B2 (2003).

12. M. L. Sentmanat, U.S. Patent No. 6,691,569 B1 (2004).

13. M. L. Sentmanat, Rheol. Acta 43, 657-669 (2004).

14. M. Zatloukal, Annual Technical Conference - ANTEC, Conference Proceedings 1, $92-96$ (2011).

15. M. Zatloukal, J. Non-Newtonian Fluid Mech. 165, 592-595 (2010).

16. H. A. Barnes and G. P. Roberts, J. Non-Newtonian Fluid Mech. 44, 113-126 (1992).

17. D. Ahirwal, S. Filipe, I. Neuhaus, M. Busch, G. Schlatter and M. Wilhelm, J. Rheol. 58, 635-658 (2014).

18. H. Münstedt and F. R. Schwarzl, Deformation and flow of polymeric materials (Springer, Heidelberg, 2014). 
AIP Conference Proceedings is copyrighted by AIP Publishing LLC (AIP). Reuse of AIP content is subject to the terms at: http://scitation.aip.org/termsconditions. For more information, see http://publishing.aip.org/authors/rights-and-permissions. 